\title{
Nonsurgical acute traumatic subdural hematoma: what is the risk?
}

\author{
Paul Bajsarowicz, MD, ${ }^{1}$ Ipshita Prakash, MD, ${ }^{2}$ Julie Lamoureux, DMD, MSc, ${ }^{3}$ \\ Rajeet Singh Saluja, MD, FRCSC, ${ }^{4}$ Mitra Feyz, MSc, ${ }^{5}$ Mohammad Maleki, MD, FRCSC, ${ }^{4,6}$ and \\ Judith Marcoux, MD, MSc, FRCSC4,6
}

\begin{abstract}
${ }^{1}$ Radiology Department, ${ }^{2}$ Department of Surgery, and ${ }^{4}$ Department of Neurology and Neurosurgery, McGill University; ${ }^{5}$ Department of Surgery and ${ }^{6}$ Department of Neurosurgery, McGill University Health Centre, Montreal, QC, Canada; and ${ }^{3}$ Baptist Health South Florida, Miami, Florida
\end{abstract}

\begin{abstract}
OBJECT The Brain Trauma Foundation has published guidelines on the surgical management of traumatic subdural hematoma (SDH). However, no data exist on the proportion of patients with SDH that can be selected for conservative management and what is the outcome of these patients. The goals of this study were as follows: 1) to establish what proportion of patients are initially treated conservatively; 2 ) to determine what proportion of patients will deteriorate and require surgical evacuation; and 3) to identify risk factors associated with deterioration and delayed surgery.
\end{abstract}

METHODS All cases of acute traumatic SDH (869 when inclusion criteria were met) presenting over a 4-year period were reviewed. For all conservatively treated SDH, the proportion of delayed surgical intervention and the Glasgow Outcome Scale score were taken as outcome measures. Multiple factors were compared between patients who required delayed surgery and patients without surgery.

RESULTS Of the 869 patients with acute traumatic SDH, 646 (74.3\%) were initially treated conservatively. A good outcome was achieved in $76.7 \%$ of the patients. Only $6.5 \%$ eventually required delayed surgery, and the median delay for surgery was 9.5 days. Factors associated with deterioration were as follows: 1) thicker SDH ( $p<0.001) ; 2)$ greater midline shift $(p<0.001) ; 3)$ location at the convexity $(p=0.001) ; 4)$ alcohol abuse $(p=0.0260)$; and 5$)$ history of falls ( $p$ $=0.018$. . There was no significant difference in regard to age, sex, Glasgow Coma Scale score, Injury Severity Score, abnormal coagulation, use of blood thinners, and presence of cerebral atrophy or white matter disease.

CONCLUSIONS The majority of patients with SDH are treated conservatively. Of those, only $6.5 \%$ later required surgery, for raised intracranial pressure or SDH progression. Patients at risk can be identified and followed more carefully. http://thejns.org/doi/abs/10.3171/2014.10.JNS141728

KEY WORDS traumatic brain injury; subdural hematoma; acute subdural hematoma; nonsurgical subdural hematoma; trauma

$\mathrm{T}$ RaUMATIC subdural hematomas (SDHs) are a common pathological entity in neurosurgical practice. Between $12 \%$ and $29 \%$ of patients admitted with a severe traumatic brain injury (TBI) have an acute SDH. When combining all patients with TBI, $11 \%$ present with an $\mathrm{SDH} .{ }^{10}$

Acute traumatic SDH has been traditionally considered a lesion that should be treated surgically, although some might be approached conservatively. In a study by
Wong, ${ }^{17}$ a midline shift greater than $5 \mathrm{~mm}$ in patients with a Glasgow Coma Scale (GCS) score below 15 was associated with deterioration and the need for surgical evacuation. In another study, ${ }^{11}$ it was the initial thickness of the SDH that was predictive of the need for surgery, with all SDHs greater than $10 \mathrm{~mm}$ in thickness requiring surgical evacuation. Based on these findings, Servadei et al. ${ }^{12}$ attempted to establish a treatment protocol for comatose patients with SDH. They selected 15 comatose patients with

ABBREVIATIONS AUC = area under the curve; $\mathrm{BCR}=$ bicaudate ratio; GCS = Glasgow Coma Scale; GOS = Glasgow Outcome Scale; ICP = intracranial pressure; ISS = Injury Severity Score; ROC = receiver operating characteristic; SDH = subdural hematoma; SFR = sylvian fissure ratio; TBI = traumatic brain injury. SUBMITTED August 4, 2014. ACCEPTED October 30, 2014.

INCLUDE WHEN CITING Published online May 8, 2015; DOI: 10.3171/2014.10.JNS141728.

DISCLOSURE The authors report no conflict of interest concerning the materials or methods used in this study or the findings specified in this paper. 
SDH for nonoperative treatment. All the selected patients had an SDH thickness of less than $10 \mathrm{~mm}$ and a midline shift of less than $5 \mathrm{~mm}$. The selected patients were also clinically stable or improving, and had no intracranial hypertension. Despite this selection, Servadei et al. found that $13 \%$ of the patients deteriorated and required delayed surgery, because of worsening intracerebral hematomas and high intracranial pressure (ICP).

The current Brain Trauma Foundation guidelines ${ }^{3}$ regarding the treatment of traumatic SDH were based on these studies. The guidelines therefore state that 1) any SDH with a thickness greater than $10 \mathrm{~mm}$ or associated with a midline shift of more than $5 \mathrm{~mm}$ on CT scans should be surgically evacuated regardless of the GCS score: ${ }^{13}$ and 2) a smaller SDH associated with a GCS score of less than 9, along with one of the following criteria-a 2-point decrease in the GCS score, an ICP higher than 20 $\mathrm{mm} \mathrm{Hg}$, or signs of herniation (pupillary asymmetry) should be surgically evacuated.

When applying these guidelines, there is to our knowledge no information in the literature on how many acute traumatic SDHs are selected for conservative management. There is also no information on how many will require an eventual surgical intervention, apart from the study by Servadei et al. ${ }^{12}$ which included only 15 patients, all of them comatose. The risk factors for such deterioration are also largely unknown.

The purposes of this study were 1) to establish what proportion of patients are initially treated conservatively; 2) to determine what proportion of patients will deteriorate and require surgical evacuation; and 3) to identify risk factors associated with deterioration.

\section{Methods \\ Patient Population}

The Montreal General Hospital, part of the McGill University Health Centre, is 1 of only 3 adult tertiary (Level 1) trauma centers serving the province of Quebec, Canada, which has a population of almost 8 million people. Based on the "Institut de la Statistique du Québec" and the Provincial Ministry of Health statistics database, the adult population served by our institution was $1,125,425$ in $20066^{6}$

\section{Selection Criteria}

The Montreal General Hospital Traumatic Brain Injury Database and the Trauma Registry Database were used to identify all patients admitted between January 1, 2006, and November 30,2009, with a diagnosis of traumatic $\mathrm{SDH}$. We performed a retrospective study of the charts and initial CT scans in all 992 patients identified, to include all those with an acute traumatic SDH. The McGill University Health Centre Ethics Review Board and the Director of Professional Services approved this study, and the informed consent requirement was waived. Patients were excluded from data collection and/or analysis for the following reasons: 1) the SDH was not traumatic; 2) the SDH was not acute; 3 ) there was no SDH (incorrectly coded); 4) patients arrived moribund and were treated only with comfort measures; or 5) charts were missing or incomplete after multiple attempts to locate them.

\section{Management of Traumatic SDH}

All patients with a traumatic SDH were initially evaluated by a trauma team, and subsequently by the neurosurgery service. Patients requiring immediate surgery according to the Brain Trauma Foundation guidelines were directed to the operating room. Therefore, patients with a symptomatic SDH greater than $10 \mathrm{~mm}$ in thickness and/or with an associated midline shift greater than $5 \mathrm{~mm}$, with decreasing GCS score or showing signs of herniation, or with increased ICP in relation to the SDH were treated surgically. According to the guidelines and to the attending neurosurgeon's clinical judgment, patients with small $\mathrm{SDH}$, patients with asymptomatic SDH, or very elderly patients with minimal symptoms were treated conservatively. All patients treated conservatively were admitted to the intensive care unit under observation. All patients with a GCS score of 8 or less and an abnormal scan had an ICP monitor placed, according to the Brain Trauma Foundation guidelines. ${ }^{2}$ Appropriate measures to lower the ICP once it exceeded $20 \mathrm{~mm} \mathrm{Hg}$ were administered as indicated, including sedation and analgesia, head of bed elevation, CSF drainage, hyperosmolar therapy, and in some cases barbiturate coma to induce a burst-suppression pattern on the electroencephalography approximately every 10 seconds and/or decompressive craniectomy. Prophylactic anticoagulation was initiated in nonambulatory patients 48-72 hours after the trauma, provided there was stability of the intracranial hemorrhagic lesions on 2 consecutive CT scans. ${ }^{5}$

\section{Data Collected}

The proportion of patients treated with urgent surgical evacuation and conservatively was calculated. The proportion of patients initially treated conservatively and later requiring a surgical intervention was then calculated, as well as the rationale for delayed surgical evacuation. Data collected as independent variables included the following: 1) demographic data: age and sex; 2) injury-related data: initial GCS score, mechanism of injury, multiple traumas versus isolated head injury, and Injury Severity Score (ISS);1 3) patient history: a history of falls, alcohol abuse, and intake of blood-thinning medication prior to admission; 4) medical information: presence of coagulopathy on initial assessment and within the first 24 hours after admission, and administration of blood-thinning medication postadmission (prophylactic anticoagulation, therapeutic anticoagulation, or antiplatelet medication); 5) any other neurosurgical interventions (excluding external ventricular drain insertion) that took place; and 6) imaging characteristics on CT scans: SDH maximum thickness, midline shift, location (convexity, parafalcial, tentorial, or posterior fossa), presence of other traumatic intracranial findings, presence of brain atrophy as calculated by the bicaudate ratio (BCR) ${ }^{4,15}$ and the sylvian fissure ratio (SFR) ${ }^{15}$ (Fig. 1), and presence of chronic white matter disease.

\section{Outcome Measures}

The primary outcome measures were as follows: 1) the percentage of conservatively treated patients who required eventual surgical evacuation of their SDH; 2) the 


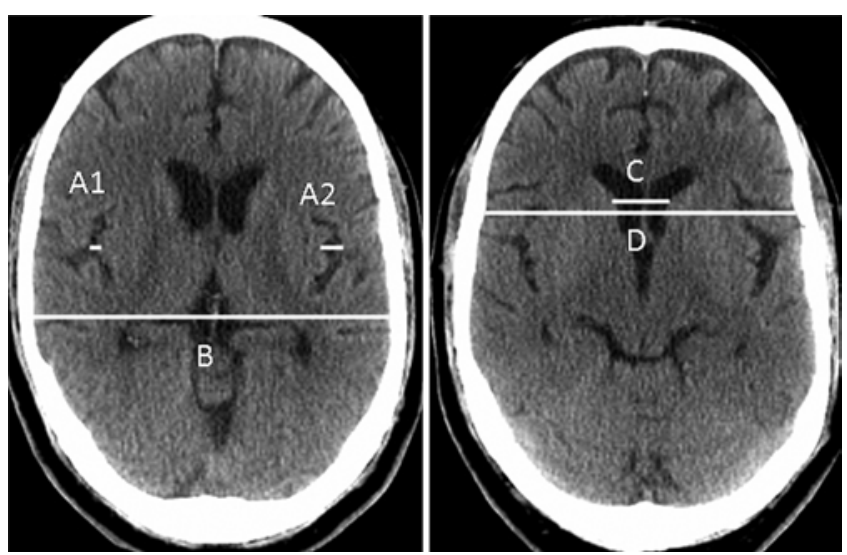

FIG. 1. The SFR and BCR as markers of brain atrophy. The SFR is the result of $(A 1+A 2) \div 2 B$. The $B C R$ is the result of $C \div D$.

timing of that delayed surgery; 3 ) the reason for this delayed surgical evacuation; and 4) the method used for surgical evacuation. Secondary outcome measures were the Glasgow Outcome Scale (GOS) score ${ }^{7,8}$ at discharge from the acute care hospital, and whether the discharge destination was home or another medical facility. The GOS score was always assigned according to a consensus within the multidisciplinary team at discharge from the acute care hospital.

\section{Statistical Analysis}

Descriptive statistics were reported for all variables as means and standard deviations for numerical variables, and as percentages for categorical data. Bivariate differences between surgery and nonsurgery groups were assessed using chi-square tests for categorical variables, t-tests for numerical data (with or without Satterthwaite correction for inequality of variances), and Wilcoxon rank-sum test for count variables or numerical variables with severe asymmetry. To determine which factors were significantly associated with a surgical intervention, a backward logistic regression was used (with the $\mathrm{p}$ value for removing variables set at $p>0.05$ ). Following logistic regression, a resulting receiver operating characteristic (ROC) curve was produced and the area under the curve (AUC) was calculated as a measure of the model's usefulness. Statistical significance was set at $\mathrm{p}<0.05$. All analyses were done using Stata version 12.1 software (StataCorp).

\section{Results}

The Montreal General Hospital's TBI and trauma registry databases contained 992 charts coded as "traumatic SDH." One hundred twenty-three patients were excluded; see Fig. 2 for the details of the exclusion process. In total, there were 869 patients treated for acute traumatic SDH during the 4-year period at our institution, and 646 $(74.3 \%)$ of them were initially treated conservatively.

\section{Need for Delayed Surgical Evacuation and Timing}

Of the 646 patients treated conservatively, only $6.5 \%$ $(\mathrm{n}=42)$ required a delayed surgical intervention to evacuate the SDH. In most cases, the reason for surgery was a progression of the SDH size with accompanying symptoms (31 of 42 patients; $73.8 \%$ ). In the remaining cases, the rationale for a surgical intervention was the development of intracranial hypertension (10 of 42 patients; $23.8 \%$ ) or status epilepticus ( 1 of 42 patients; $2.4 \%$ ). Nine of the 10 patients requiring intervention for intracranial hypertension underwent large decompressive craniectomy along with SDH evacuation. One patient had a massive sudden rehemorrhage 15 days after an initial small (6-mm maximum thickness, no midline shift) SDH. This particular patient died before having a surgical intervention. Therefore, $5 \%$ of conservatively treated patients experienced significant rebleeding (needing surgery or resulting in death). The delay before surgery was asymmetrically distributed, with a median of 9.5 days, an interquartile range between 4 and 18 days, and a total range between 1 and 170 days. Ninety percent of the patients had their surgery within 43 days of the trauma. Those who underwent surgery for an increased ICP were operated on earlier, with a median time of 3.5 days compared with 14 days for those who had a progression of their SDH (Wilcoxon rank-sum test; $\mathrm{z}=3.698, \mathrm{p}<0.001)$. Figure 3 gives the distribution of the delay until surgery.

\section{Factors Associated With the Need for Delayed Surgical Intervention}

Bivariate associations between the occurrence of late surgery in patients who originally were conservatively treated and predisposing factors were investigated. Table 1 lists all the factors, the statistical tests used for each, and the associated $\mathrm{p}$ values.

Factors significantly associated with failed conservative treatment include history of alcohol abuse, history of multiple falls, and thickness of the main SDH (in the cases where there was more than one location, the largest one was used). Figure 4 shows the distribution of the thickness of the main SDH for the whole sample. Similarly, the midline shift was significantly greater in the surgery group compared with the nonsurgery group. The location of the main SDH was also significantly different between the groups; in $100 \%$ of all patients with a delayed surgery the main SDH was located in the convexity.

There was no age difference between the group of patients who had surgery and the group without surgery. On the other hand, there was a significant age difference (Wilcoxon rank-sum test; $\mathrm{z}=4.196, \mathrm{p}<0.001$ ) between those who had a delayed surgery for progression of their SDH $(68.8 \pm 14.4)$ compared with those who had high ICP $(36.7 \pm 13.6)$. There were no sex differences between the 2 groups. The mechanism of trauma was not significantly different between the groups either; in both groups, the most common mechanism of accident was fall from the patient's own height. The initial GCS and the ISS values were not significantly different between the 2 groups. The proportion of multiple traumas was not significantly different between the groups either, and there were no significant differences in the use of blood thinner before or after the trauma.

In the group that required surgical intervention, 52.4\% received venous thromboembolic prophylaxis within 3 days of the admission, compared with $44 \%$ of the group 


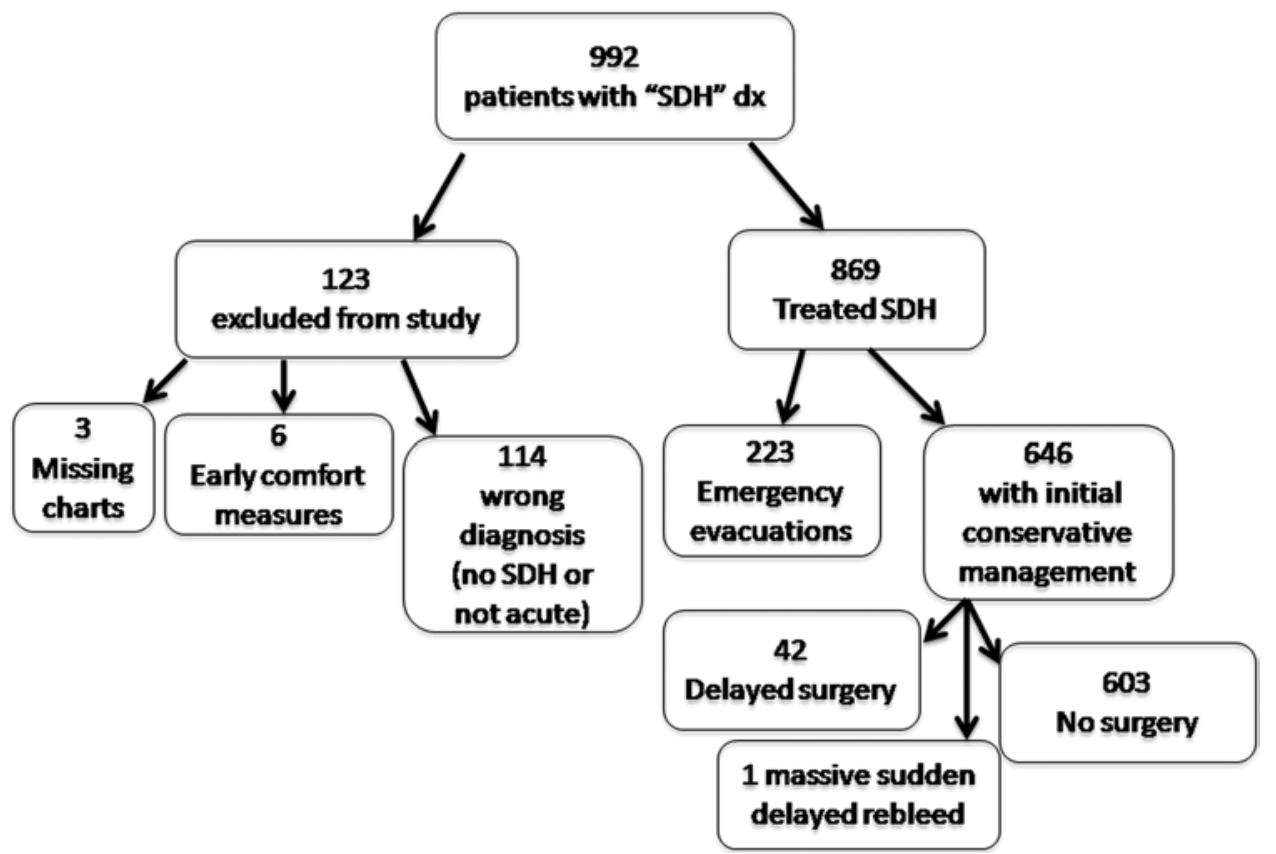

FIG. 2. Flowchart of the selection process, showing how patients with an initial diagnosis (dx) of traumatic SDH were excluded from the study.

that did not require surgery. No patient in the surgery group received therapeutic anticoagulation, compared with $2.2 \%$ of the patients in the nonsurgical group. Therapeutic anticoagulation was initiated on average 10 days after the admission. Antiplatelet therapy was initiated in $2.4 \%$ of the surgical group patients, compared with $5 \%$ of the nonsurgical group patients, with a mean delay of 10 days postadmission. There was no significant difference in the rate of abnormal coagulation between the 2 groups.

Although none of the patients in this sample had early surgery for their SDHs, some had other intracranial interventions like repair of depressed skull fractures, craniotomy for evacuation of epidural hematoma, or intraparenchymal hematoma, and so on. The percentage of patients

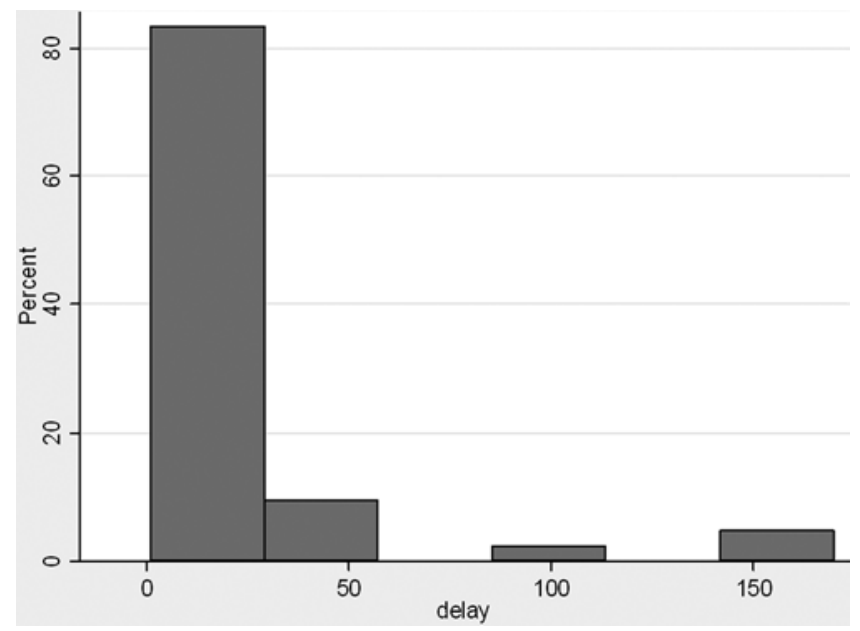

FIG. 3. Bar graph showing the delay, which represents the number of days after trauma at which a surgical intervention became necessary. The percent represents the percentage of patients requiring intervention for each time period. Total number $=42$. having undergone previous intracranial interventions in the group with delayed SDH surgery and in the group without delayed surgery was not significantly different. The percentage of patients with more than one SDH was not significantly different between the groups. Also, the percentage with white matter disease was not significantly different between the groups. Both the SFR and the BCR showed a slight asymmetry in their distribution (Fig. 5). The SFR score was not significantly different between the groups. The BCR score was not significantly different between the groups either, although it did approach statistical significance $(\mathrm{p}=0.052)$.

\section{Prediction of Delayed Surgery}

A multiple logistic regression was done to determine which of the measured variables could help predict the need for delayed surgery in patients with SDH. Using the variables that were significant at $p<0.20$ in the bivariate associations described above (age, GCS score, previous fall, history of alcohol abuse, multiple traumas, location of main SDH, thickness of SDH, number of SDHs, and BCR), we performed backward elimination to obtain a predictive model containing only variables significantly associated with the outcome at the $\mathrm{p}<0.05$ level of significance. Because midline shift is a criterion often used by itself to determine the need for delayed surgery, we first compared the AUC for a model containing only midline shift as a predictor with the AUC for a model containing other significant predictors. This allowed us to compare the relative usefulness of the 2 models, and then a combined model was done to create the most complete predictive model.

The model containing only "midline shift" as a predictive variable had an AUC for the ROC curve of 0.772. The odds ratio associated with midline shift was 1.60 , indicat- 
TABLE 1. Comparison of the groups' characteristics in 645 patients with and without delayed surgery*

\begin{tabular}{|c|c|c|c|c|}
\hline Variable & No Surgery $(n=603)$ & Surgery $(n=42)$ & Test, Results & p Value \\
\hline Age (yrs); mean \pm SD & $56.1 \pm 22.7$ & $60.8 \pm 19.5$ & $t_{(643 \mathrm{df})}=1.303$ & 0.193 \\
\hline Sex (\% males) & $69.8 \%$ & $71.4 \%$ & $\chi_{1 \mathrm{df}}^{2}=0.049$ & 0.826 \\
\hline Median GCS score, IQR & $14,13-15$ & $14,8-15$ & $z=1.910$ & 0.056 \\
\hline ISS; mean \pm SD & $26.0 \pm 9.0$ & $26.8 \pm 7.7$ & $z=0.747$ & 0.455 \\
\hline Previous fall & $24.1 \%$ & $40.5 \%$ & $\chi_{1 d f}^{2}=5.575$ & $0.018 \dagger$ \\
\hline History of alcohol abuse & $27.7 \%$ & $43.9 \%$ & $\chi_{1 \mathrm{df}}^{2}=4.935$ & $0.026 \dagger$ \\
\hline Multiple trauma & $45.3 \%$ & $33.3 \%$ & $\chi_{1 \text { dff }}^{2}=2.267$ & 0.132 \\
\hline Preadmission blood thinner use & & & $\chi_{3 \mathrm{df}}^{2}=3.535$ & 0.316 \\
\hline None & $68.7 \%$ & $59.5 \%$ & & \\
\hline Platelet & $26.0 \%$ & $28.6 \%$ & & \\
\hline Anticoag & $3.0 \%$ & $7.1 \%$ & & \\
\hline Both & $2.3 \%$ & $4.8 \%$ & & \\
\hline Postadmission blood thinner use & & & $\chi_{4 \mathrm{df}}^{2}=5.171$ & 0.270 \\
\hline None & $52.8 \%$ & $45.2 \%$ & & \\
\hline Prophylactic anticoag & $40.0 \%$ & $52.4 \%$ & & \\
\hline Platelet \& prophylactic anticoag & $4.0 \%$ & $0.0 \%$ & & \\
\hline Therapeutic anticoag & $2.2 \%$ & $0.0 \%$ & & \\
\hline Platelet & $1.0 \%$ & $2.4 \%$ & & \\
\hline Abnormal coagulation & $14.9 \%$ & $21.4 \%$ & $\chi_{1 \mathrm{df}}^{2}=1.273$ & 0.259 \\
\hline Previous intracranial surgery & $5.7 \%$ & $7.1 \%$ & $\chi_{1 d f}^{2}=0.162$ & 0.687 \\
\hline Location of main SDH & & & $\chi_{3 \mathrm{df}}^{2}=15.730$ & $0.001 \dagger$ \\
\hline Convexity & $72.2 \%$ & $100.0 \%$ & & \\
\hline Parafalcial & $15.0 \%$ & $0.0 \%$ & & \\
\hline Tentorial & $12.5 \%$ & $0.0 \%$ & & \\
\hline Suboccipital & $0.3 \%$ & $0.0 \%$ & & \\
\hline Thickness $(\mathrm{mm})$ of SDH; mean \pm SD & $5.4 \pm 3.4$ & $8.1 \pm 3.9$ & $z=4.786$ & $<0.001 \dagger$ \\
\hline Midline shift $(\mathrm{mm}) ;$ mean $\pm \mathrm{SD}$ & $0.4 \pm 1.3$ & $3.3 \pm 3.5$ & $z=9.102$ & $<0.001 \dagger$ \\
\hline No. of SDHs & & & $\chi_{2 \mathrm{df}}^{2}=3.394$ & 0.183 \\
\hline 1 & $84.1 \%$ & $76.2 \%$ & & \\
\hline 2 & $14.3 \%$ & $23.8 \%$ & & \\
\hline 3 & $1.7 \%$ & $0.0 \%$ & & \\
\hline White matter disease & $20.1 \%$ & $14.3 \%$ & $\chi_{\text {1df }}^{2}=0.830$ & 0.362 \\
\hline SFR; mean \pm SD & $0.06 \pm 0.03$ & $0.06 \pm 0.02$ & $z=0.795$ & 0.427 \\
\hline $\mathrm{BCR} ;$ mean $\pm \mathrm{SD}$ & $0.12 \pm 0.04$ & $0.11 \pm 0.04$ & $z=1.947$ & 0.052 \\
\hline
\end{tabular}

Anticoag = anticoagulant; IQR = interquartile range; ISS = Injury Severity Score.

* Values are expressed as percentages unless otherwise indicated. There were 646 patients in all, but 1 individual had a massive, sudden rehemorrhage 15 days after the initial $\mathrm{SDH}$; this patient died before having a surgical intervention.

$\dagger$ Statistically significant.

ing that for every 1-mm increase in the midline shift, the odds of surgery increased by $66 \%$. The final model containing the other predictive variables is described in Table 2. The AUC for the ROC curve for this model was 0.780 , which is similar to that of the univariate model containing only midline shift. In this model, a history of alcohol abuse doubles the odds of delayed surgery. We also see that for every increase of $1 \mathrm{~mm}$ in the thickness of the SDH, the odds of surgery increased by $16 \%$. If we combine the 2 models, in the resulting model (described in Table 3) the AUC climbs to 0.855 , indicating that the model containing more than the midline shift is a better predictor of delayed surgery than the one with the midline shift variable alone.

\section{Outcome at Discharge}

In total, $76.7 \%$ of the patient cohort achieved a good outcome (GOS Score 4 or 5). An ordinal logistic regression was done to determine the odds of having a better outcome (a higher score on the GOS) whether or not the patients had delayed surgery. Those who had delayed surgery were approximately 2 times less likely (OR 0.488) to have a better outcome than those without delayed surgery. The percentage of deaths is similar in the 2 groups, but there seems to be a higher proportion of patients with severe disability in the group with surgery. None of the patients in the delayed surgery group had a GOS score of 5 at discharge. On the other hand, the only 5 patients who 


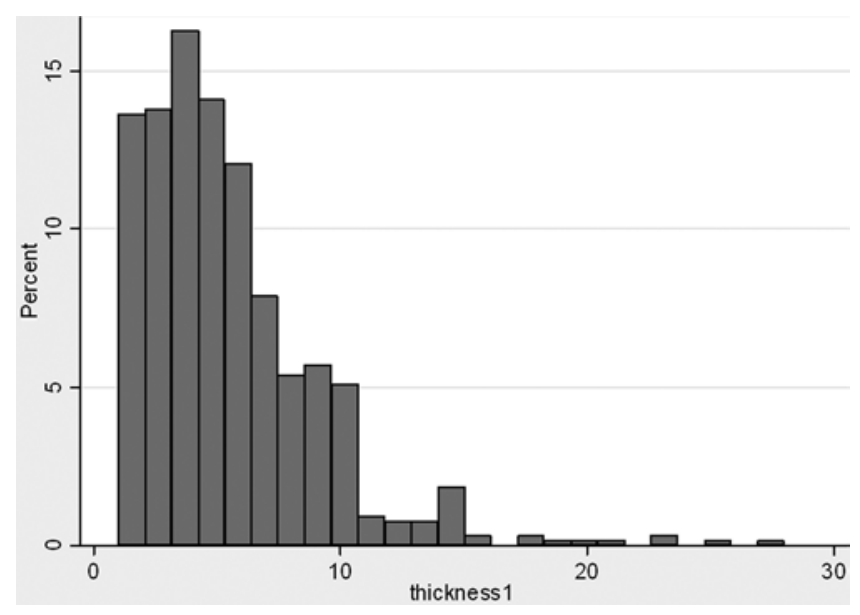

FIG. 4. Bar graph showing distribution of the thickness of the main SDH (thickness1, expressed in millimeters) for the whole sample, as a percentage.

were discharged in a vegetative state were in the nonsurgery group. Table 4 depicts the outcome according to the GOS score for each group.

\section{Discussion}

Our study is the first to look at the outcome of nonsurgically treated traumatic SDH since the publication and application of the Brain Trauma Foundation guidelines. It shows that the majority of acute traumatic SDH cases are actually initially treated conservatively, with a proportion of $74.3 \%$. Our study also shows that for this large group of patients, only $6.5 \%$ experienced a deterioration of their condition that required a surgical intervention. For most patients $(73.8 \%)$, the late intervention was due to an increase in the SDH size over time, whereas for the remaining patients an increasing ICP $(23.8 \%)$ or status epilepticus (2.4\%) led to the surgical intervention.

The deterioration occurred at a median of 9.5 days after the trauma, yet in many instances the deterioration occurred in a very delayed fashion (months). Bivariately, the risk factors of deterioration were the measures associated with the SDH size and mass effect (larger SDH and greater midline shift), the history of repetitive falls, alcohol abuse, and lesion location at the convexity. The finding that larger SDHs with worse mass effect are at higher risk correlates with the results from the studies by Mathew et al. ${ }^{11}$ and Wong, ${ }^{17}$ and is most probably due to the fact that there is less room for any deterioration, compared with a small hematoma without mass effect. The repetitive falls can play a major role in the delayed progression of the SDH, from repeated small traumas causing new hemorrhages. The fact that convexity SDHs are the only ones that required delayed surgery, and that none of the SDHs in a parafalcial,
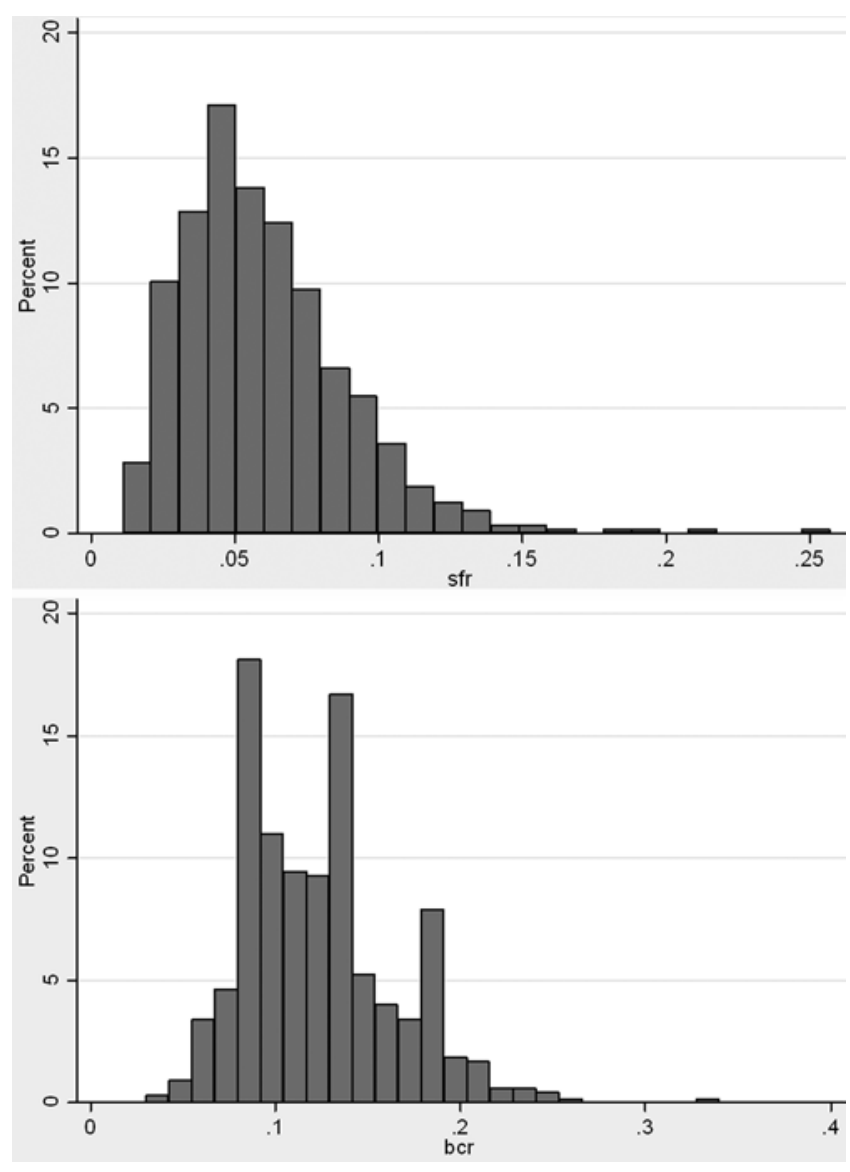

FIG. 5. Bar graphs showing distribution of the SFR (upper) and the BCR (lower), in percentage. Both the SFR and the BCR show a slight asymmetry in their distribution.

tentorial, or posterior fossa location progressed, is harder to explain. Delayed progression of $\mathrm{SDH}$ and formation of chronic SDH are thought to come from recurrent bleeding from the hematoma capsule. It is also believed that exudation from the macrocapillaries in the outer membrane plays an important role in the enlargement of hematoma. ${ }^{14}$ It is not clear why those processes would be different for parafalcial and tentorial SDH, although a smaller number of bridging veins and less tension on them may explain the difference. Furthermore, the fact that there is less counterpressure from the brain in the convexity could predispose to the growth of SDH in that particular location. ${ }^{9}$

Brain atrophy was not found to be an independent risk factor of SDH progression in our study. This finding is in contradiction with reports in the literature on the development of chronic SDH. Indeed, cerebral atrophy was found to be associated with the development of chronic SDH. ${ }^{16}$ It is possible that our calculations of cerebral atrophy through indirect measures (CSF space rather than

TABLE 2. Predictive model for delayed surgery using baseline variables

\begin{tabular}{llllll}
\hline Surgery & OR & SE & $z$ & $P>|z|$ & $95 \% \mathrm{Cl}$ \\
\hline Alcohol & 2.204259 & 0.744563 & 2.34 & 0.019 & $1.136948-4.27351$ \\
\hline Thickness & 1.164174 & 0.0397847 & 4.45 & 0.000 & $1.088751-1.244821$ \\
\hline Constant & 0.0198315 & 0.0068496 & -11.35 & 0.000 & $0.010077-0.0390258$ \\
\hline
\end{tabular}


TABLE 3. Predictive model for delayed surgery using baseline variables and midline shift

\begin{tabular}{lccccc}
\hline \multicolumn{1}{c}{ Surgery } & OR & SE & $z$ & $P>|z|$ & $95 \% \mathrm{Cl}$ \\
\hline Alcohol & 2.480773 & 0.9275853 & 2.43 & 0.015 & $1.192105-5.162494$ \\
\hline Thickness & 1.099525 & 0.0446011 & 2.34 & 0.019 & $1.015493-1.190511$ \\
\hline Midline shift & 1.601998 & 0.1129809 & 6.68 & 0.000 & $1.395182-1.839471$ \\
\hline Constant & 0.0138366 & 0.005625 & -10.53 & 0.000 & $0.0062372-0.0306953$ \\
\hline
\end{tabular}

cerebral volume) might have been underestimations due to the space-occupying SDH.

The administration of blood thinners was also not associated with an increased risk of deterioration. Bloodthinning medication was used in approximately half of the patients in both groups, and the blood-thinning medication most used was subcutaneous heparin or low-molecularweight heparin at prophylactic doses. Very few patients received therapeutic anticoagulation or antiplatelet therapy, and this low frequency may be an explanation for the lack of statistical significance.

When all the factors were considered together, the most significant predictors of late surgery in this population were midline shift, thickness of the main SDH, convexity location, alcohol abuse, and repetitive falls. The results of a predictive model with promising sensitivity and specificity point to the possibility of creating an algorithm that would help predict the risk associated with late surgery.

A good outcome was achieved in $77 \%$ of all patients who were initially treated conservatively. The GOS score was lower overall for those who eventually required surgery. This difference might be explained by the fact that these patients had a surgical intervention and had a larger hematoma. However, the GCS and ISS values were similar, suggesting that the 2 groups did not differ significantly initially in the severity of their trauma.

Although the Brain Foundation's surgical guidelines were followed, the surgical group had a mean SDH thickness of $8.1 \mathrm{~mm}$. The majority of hematomas measuring more than $10 \mathrm{~mm}$ were seen in very elderly patients with little or no mass effect from those large hematomas. Another subset of patients with larger lesions had only focally large hematomas, and therefore had few or no associated symptoms. Those larger SDHs were therefore observed rather than surgically treated.

Smaller SDHs were also found on occasion to require surgical intervention, often in a very delayed fashion. These small SDHs were clearly not surgical candidates at

TABLE 4. Comparison of the groups' outcome with and without delayed surgery

\begin{tabular}{lrrrr}
\hline \multicolumn{1}{c}{ Variable } & $\begin{array}{c}\text { No Surgery } \\
(n=603)\end{array}$ & $\begin{array}{c}\text { Surgery } \\
(n=42)\end{array}$ & $\begin{array}{c}\text { Test, } \\
\text { Results }\end{array}$ & p Value \\
\hline GOS score & & & OR $=0.488$ & $0.021^{*}$ \\
\hline Death & $8.0 \%$ & $9.5 \%$ & & \\
\hline Vegetative state & $0.8 \%$ & $0.0 \%$ & & \\
\hline Severe disability & $13.4 \%$ & $28.6 \%$ & & \\
\hline Moderate disability & $73.8 \%$ & $61.9 \%$ & & \\
\hline Normal state & $4.0 \%$ & $0.0 \%$ & & \\
\hline * Statistically significant & & &
\end{tabular}

presentation, yet did not evolve favorably by spontaneous resolution. Instead, they expanded, rehemorrhaged, and became chronic in nature and larger with time. They do not represent failed conservative treatment per se, and the operative treatment of the chronic SDH might have been a less morbidity-producing procedure than early evacuation of a very small acute SDH.

This study had some drawbacks. As with all retrospective studies, some data could have been missing or misinterpreted. A few charts could not be completed (missing parts or unavailable imaging). Some late deterioration could have been missed if the patient presented at another hospital or, worse, died. However, our institution is a Level 1 trauma center and all patients with traumatic injuries are referred to us, and followed by us, so only a very few cases might have been missed. Furthermore, the very large number of patients included should attenuate the effect of missing information in a few cases. Functional outcome was evaluated early in the evolution of the patients' lesions, and using only the 5-point GOS scoring system. Unfortunately, only the outcome at discharge is systematically calculated and recorded for all patients. This short-term outcome can underestimate the functional recovery of some of the patients in the long term.

\section{Conclusions}

Most acute traumatic SDHs are managed conservatively, with $77 \%$ of the patients in this study achieving a good early outcome. Only a small proportion of the conservatively managed patients will deteriorate and require surgery. Patients with a larger SDH, a lesion located at the convexity, alcohol abuse, and repetitive falls have the highest risk for deterioration. Surveillance is recommended until resolution of the $\mathrm{SDH}$, or at least up to 5 weeks after the trauma.

\section{References}

1. Baker SP, O’Neill B, Haddon W Jr, Long WB: The injury severity score: a method for describing patients with multiple injuries and evaluating emergency care. J Trauma 14:187196, 1974

2. Bratton SL, Chestnut RM, Ghajar J, McConnell Hammond FF, Harris OA, Hartl R, et al: Guidelines for the management of severe traumatic brain injury. VI. Indications for intracranial pressure monitoring. J Neurotrauma 24 (Suppl 1):S37S44, 2007

3. Bullock MR, Chesnut R, Ghajar J, Gordon D, Hartl R, Newell DW, et al: Surgical management of acute subdural hematomas. Neurosurgery 58 (3 Suppl):S16-S24, Si-iv, 2006

4. Doraiswamy PM, Patterson L, Na C, Husain MM, Boyko O, McDonald WM, et al: Bicaudate index on magnetic resonance imaging: effects of normal aging. J Geriatr Psychiatry Neurol 7:13-17, 1994 
5. Dudley RR, Aziz I, Bonnici A, Saluja RS, Lamoureux J, Kalmovitch B, et al: Early venous thromboembolic event prophylaxis in traumatic brain injury with low-molecular-weight heparin: risks and benefits. J Neurotrauma 27:2165-2172, 2010

6. Institut de la Statistique du Québec: Projections de la population du Québec selon le territoire de réseau local de services (RLS), le sexe, l'âge et le groupe d'âge, 2006 à 2031. (http://www.informa.msss.gouv.qc.ca/Details. aspx?Id=vcuCWZHK870=) [Accessed March 23, 2015]

7. Jennett B, Bond M: Assessment of outcome after severe brain damage. Lancet 1:480-484, 1975

8. Jennett B, Snoek J, Bond MR, Brooks N: Disability after severe head injury: observations on the use of the Glasgow Outcome Scale. J Neurol Neurosurg Psychiatry 44:285293, 1981

9. Lee KS, Bae WK, Doh JW, Bae HG, Yun IG: Origin of chronic subdural haematoma and relation to traumatic subdural lesions. Brain Inj 12:901-910, 1998

10. Massaro F, Lanotte M, Faccani G, Triolo C: One hundred and twenty-seven cases of acute subdural haematoma operated on. Correlation between CT scan findings and outcome. Acta Neurochir (Wien) 138:185-191, 1996

11. Mathew P, Oluoch-Olunya DL, Condon BR, Bullock R: Acute subdural haematoma in the conscious patient: outcome with initial non-operative management. Acta Neurochir (Wien) 121:100-108, 1993

12. Servadei F, Nasi MT, Cremonini AM, Giuliani G, Cenni P, Nanni A: Importance of a reliable admission Glasgow Coma Scale score for determining the need for evacuation of posttraumatic subdural hematomas: a prospective study of 65 patients. J Trauma 44:868-873, 1998
13. Teasdale G, Jennett B: Assessment of coma and impaired consciousness. A practical scale. Lancet 2:81-84, 1974

14. Tokmak M, Iplikcioglu AC, Bek S, Gökduman CA, Erdal M: The role of exudation in chronic subdural hematomas. J Neurosurg 107:290-295, 2007

15. van Zagten M, Kessels F, Boiten J, Lodder J: Interobserver agreement in the assessment of cerebral atrophy on CT using bicaudate and sylvian-fissure ratios. Neuroradiology 41:261264, 1999

16. Yang AI, Balser DS, Mikheev A, Offen S, Huang JH, Babb $\mathrm{J}$, et al: Cerebral atrophy is associated with development of chronic subdural haematoma. Brain Inj 26:1731-1736, 2012

17. Wong CW: Criteria for conservative treatment of supratentorial acute subdural haematomas. Acta Neurochir (Wien) 135:38-43, 1995

\section{Author Contributions}

Conception and design: Marcoux, Saluja, Maleki. Acquisition of data: Marcoux, Bajsarowicz, Prakash, Feyz. Analysis and interpretation of data: Marcoux. Drafting the article: Marcoux, Bajsarowicz. Critically revising the article: Marcoux, Lamoureux, Saluja. Reviewed submitted version of manuscript: Marcoux. Approved the final version of the manuscript on behalf of all authors: Marcoux. Statistical analysis: Lamoureux. Study supervision: Marcoux, Saluja.

\section{Correspondence}

Judith Marcoux, Department of Neurosurgery, Montreal General Hospital, McGill University Health Centre, 1650 Cedar Ave., Rm. L7-516, Montreal, QC H3G 1A4, Canada. email: judith. marcoux@mcgill.ca. 